\title{
A Fuzzy-based Handover System for Avoiding Ping-Pong Effect in Wireless Cellular Networks
}

\author{
Leonard Barolli ${ }^{\dagger}$, Fatos Xhafa ${ }^{\ddagger}$, Arjan Durresi ${ }^{\dagger \ddagger}$, Akio Koyama ${ }^{\ddagger \dagger}$ \\ ${ }^{\dagger}$ Department of Information and Communication Engineering \\ Fukuoka Institute of Technology (FIT) \\ 3-30-1 Wajiro-Higashi, Higashi-Ku, Fukuoka 811-0295, Japan \\ E-mail: barolli@fit.ac.jp \\ ${ }^{\ddagger}$ Department of Languages and Informatics Systems, \\ Polytechnic University of Catalonia \\ Jordi Girona 1-3, 08034 Barcelona, Spain \\ E-mail: fatos@1si.upc.edu \\ $\dagger \ddagger$ Department of Computer and Information Science \\ Indiana University Purdue University at Indianapolis \\ 723 W. Michigan Street SL 280, Indianapolis, IN 46202, USA \\ E-mail: durresi@cs.iupui.edu \\ $\ddagger^{\dagger}$ Department of Informatics, Yamagata University \\ 4-3-16 Jonan, Yonezawa 992-8510, Yamagata, Japan \\ E-mail: akoyama@eie.yz.yamagata-u.ac.jp
}

\begin{abstract}
Many handover algorithms are proposed in the literature. However, to make a better handover and keep the QoS in wireless networks is very difficult. In this paper, we propose a new handover system based on fuzzy logic. The proposed system uses 3 parameters for handoff decision: the change of signal strength of the present Base Station (BS), signal strength from the neighbor BS, and the distance between Mobile Station (MS) and BS. The performance evaluation via simulations shows that proposed system can avoid ping-pong effect and has a good handover decision.
\end{abstract}

\section{Introduction}

During the last few years wireless multimedia networks have been a very active research area [1,2]. The QoS support for future wireless networks is a very important problem. To guarantee the QoS, a good handover strategy is needed in order to balance the call blocking and call dropping for providing the required QoS $[3,4]$. In the future, the wireless networks will adopt a micro/pico cellular architecture. However, smaller cell size naturally increases the number of handoffs a Mobile Station (MS) is expected to make $[5,6]$.
Many metrics have been used to support handover decisions, including Received Signal Strength (RSS), Signal to Interference Ratio (SIR), distance between the mobile and BS, traffic load, and mobile velocity, where RSS is the most commonly used one. The conventional handover decision compares the RSS from the serving BS with that from one of the target BSs, using a constant handover threshold value (handover margin). However, the fluctuations of signal strength associated with shadow fading cause the pingpong effect [7].

Many investigations have addressed handover algorithms for cellular communication systems. However, it is essentially complex to make handover decision considering multiple criteria. Sometimes, the trade-off of some criteria should be considered. Therefore, heuristic approaches based on Neural Networks (NN), Genetic Algorithms (GA) and Fuzzy Logic (FL) can prove to be efficient for wireless networks $[8,9,10,11]$. In [10], a multi-criteria handover algorithm for next generation tactical communication systems is introduced. The handover metrics are: RSS from current and candidate base transceivers, ratio of used soft capacity to the total soft capacity of base transceivers, the relative directions and speeds of the base transceivers and the mobile node. In [11], a handover algorithm is proposed to support vertical handover between heterogeneous networks. This is 
achieved by incorporating the mobile IP principles in combination with FL concepts utilizing different handover parameters.

In this paper, in different from other works we use Random Walk (RW) model and FL to design a new handover system, which is able to avoid ping-pong effect and has a good handover decision. The structure of this paper is as follows. In Section 2, we present the handover decision problem. In Section 3, we give a brief introduction of RW model. In Section 4, we introduce the proposed system. In Section 5, we discuss the simulation results. Finally, some conclusions are given in Section 6.

\section{Handover Decision Problem}

Handoffs which are consistently both accurate and timely can result in higher capacity and better overall link quality than what is available with today systems $[12,13]$. Now with increasing demands for more system capacity, there is a trend toward smaller cells, also known as microcells. Handoffs are more critical in systems with smaller cells, because for a given average user speed, handoff rates tend to be inversely proportional to cell size [5].

The main objectives of handover are link quality maintenance, interference reduction and keeping the number of handoffs low. Also, a handover algorithm should initiate a handoff if and only if the handoff is necessary. The accuracy of a handover algorithm is based on how the algorithm initiates the handover process. The timing of the handoff initiation is also important. There can be deleterious effects on link quality and interference if the initiation is too early or too late. A timely handover algorithm is one which initiates handoffs neither too early nor too late.

Because of large-scale and small-scale fades are frequently encountered in mobile environment, it is very difficult for handover algorithm to make an accurate and timely decision. Handover algorithms operating in real time have to make decisions without the luxury of repeated uncorrelated measurements or the future signal strength information. It should be noted that some of handover criteria information can be inherently imprecise, or the precise information is difficult to obtain. For this reason, we propose a FL-based approach, which can operate with imprecision data and can model nonlinear functions with arbitrary complexity.

\section{RW Model}

The Monte Carlo (MC) method is a technique that uses random numbers and probability to solve problems. It is often used when the model is complex, nonlinear, or involves more than just a couple uncertain parameters.

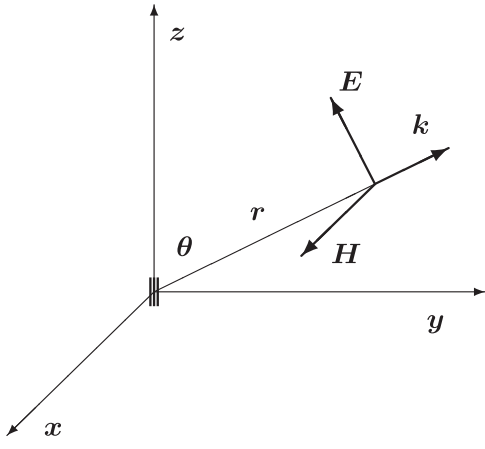

Figure 1. Dipole antenna.

The MC method can be used for analyzing uncertainty propagation, where the goal is to determine how random variation, lack of knowledge, or error affects the sensitivity, performance, or reliability of the system that is being modeled. MC simulation is categorized as a sampling method because the inputs are randomly generated from probability distributions to simulate the process of sampling from an actual population. The data generated from the simulation can be represented as probability distributions (or histograms) or converted to error bars, reliability predictions, tolerance zones, and confidence intervals.

We use the MC method for realizing RW model. We consider a 2-dimensional field. The initial position is considered as a origin point and we decided based on MC method the moving pattern for each walk. If we consider $n$ user movements and the angle $\theta$ and distance $d$ for each walk are generated by general or Gaussian distribution, when the movement changes in $x$ and $y$ directions are $\Delta x$ and $\Delta y$, respectively, then we have the following relations.

$$
\begin{gathered}
\Delta x_{n}=d_{n} \cos \theta_{n}, \quad \Delta y_{n}=d_{n} \sin \theta_{n} \\
x_{n+1}=x_{n}+\Delta x_{n}, \quad y_{n+1}=y_{n}+\Delta y_{n}
\end{gathered}
$$

The Base Station (BS) position can be expressed by Cartesian coordinates. By converting Cartesian coordinates to polar ones, we can calculate the angle $\theta$.

We consider that in the cellular system each cell has a hexagonal shape and the BS is located in the center of the cell. The angle $\theta$ between Dipole Antenna (DA) and vector $\boldsymbol{r}$ is $D(\theta)=\sin \theta$. If we consider the transmission power as $W$, the antenna radiation intensity can be calculated as follows:

$$
\boldsymbol{E}=\sqrt{45 W} \sin \theta \frac{e^{-j \kappa r}}{r^{n}} \boldsymbol{u}_{\mathbf{0}}
$$

where, the DA gain is $G=1.5$ and $\boldsymbol{u}_{0}$ is the unit vector that shows DA direction. In Fig. $1 \boldsymbol{u}_{0}$ is in $Z$ direction. 


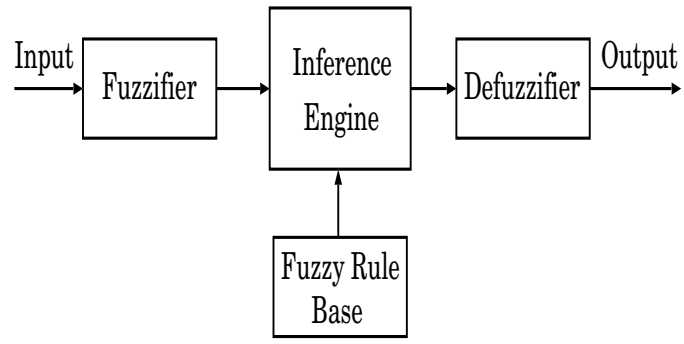

Figure 2. FLC structure.
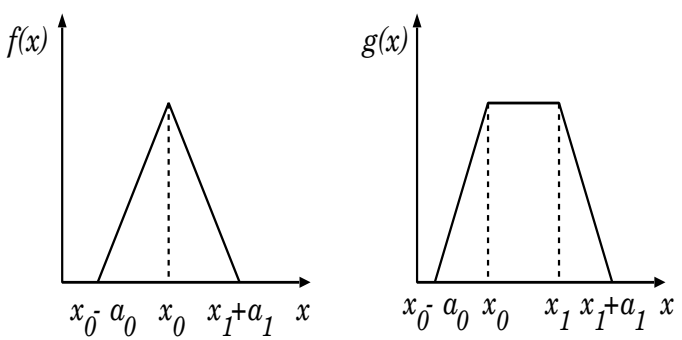

Figure 3. Membership function shapes.

In Eq.(3), when $\theta=90^{\circ}$, the $E$ value will be maximal in horizontal direction. However, in real situations, the direction of antenna is in not set $90^{\circ}$ in order to cover better the cell area. If we consider the beam tilting angle and the distance, the $E$ can be calculated by the following equation.

$$
\boldsymbol{E}=\sqrt{45 W} \sin (\theta-\phi) \frac{e^{-j \kappa r}}{r^{n}} \boldsymbol{u}_{\mathbf{0}}
$$

\section{Proposed System Model}

The Fuzzy Logic Controller (FLC) is the main part of the proposed system and its basic elements are shown in Fig. 2. They are the fuzzifier, inference engine, Fuzzy Rule Base (FRB) and defuzzifier. As shown in Fig. 3, as membership functions we use triangular and trapezoidal membership functions because they are suitable for real-time operation [14].

In Fig. 3, $x_{0}$ in $f($.$) is the center of triangular function,$ $x_{0}\left(x_{1}\right)$ in $g($.$) is the left (right) edge of trapezoidal func-$ tion, and $a_{0}\left(a_{1}\right)$ is the left (right) width of the triangular or trapezoidal function.

The proposed fuzzy model is shown in Fig. 4. In this system, the $N_{0} e_{-} B$ shows the wireless transmitter and receiver of BS, RNS indicates Radio Network System, POTLC stands for Post Test-Loop Controller and PRTLC for Pre Test-Loop Controller.

The input parameters for FLC are: Change of the Signal Strength of Present BS (CSSP), Signal Strength from the Neighbor BS $(S S N)$, and the distance of MS from BS

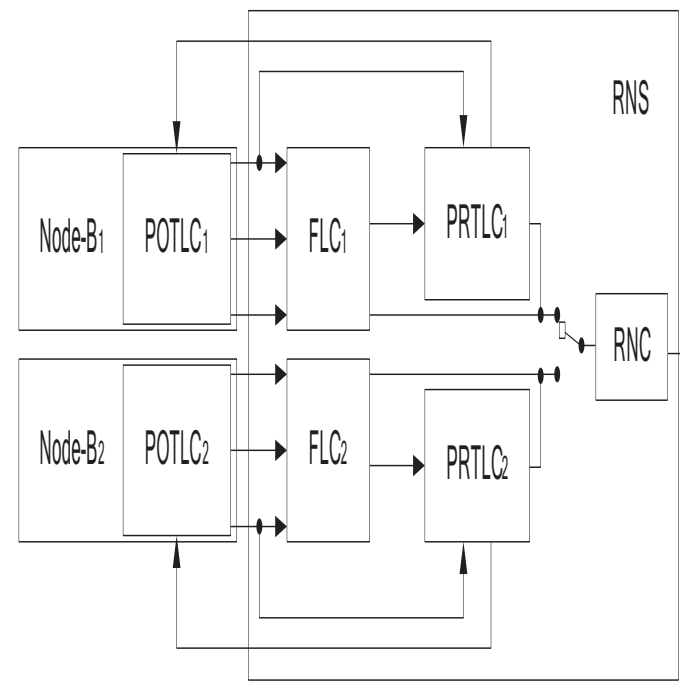

Figure 4. System model.
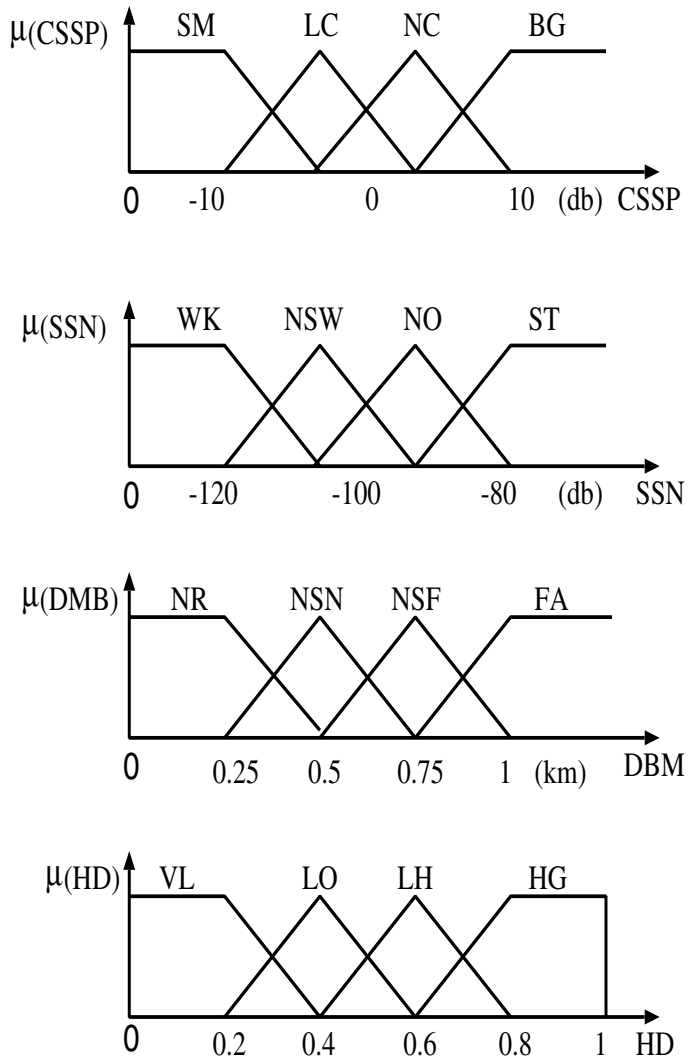

Figure 5. Membership functions.

$(D M B)$, while the output linguistic parameter is Handover Decision $(H D)$. 
Table 1. FRB.

\begin{tabular}{|c|c|c|c|c|c|c|c|c|c|}
\hline Rules & CSSP & SSN & DMB & HD & Rules & CSSP & SSN & DMB & HD \\
\hline 1 & SM & $\overline{\text { WK }}$ & NR & $\overline{\mathrm{LO}}$ & 33 & $\mathrm{NC}$ & $\overline{\mathrm{WK}}$ & NR & $\overline{\mathrm{VL}}$ \\
\hline 2 & $\overline{\mathrm{SM}}$ & WK & NSN & $\overline{\mathrm{LO}}$ & 34 & $\mathrm{NC}$ & WK & NSN & $\overline{\mathrm{VL}}$ \\
\hline 3 & SM & WK & NSF & LH & 35 & $\mathrm{NC}$ & WK & $\mathrm{NSF}$ & VL \\
\hline 4 & SM & WK & FA & LH & 36 & $\mathrm{NC}$ & WK & FA & LO \\
\hline 5 & $\overline{S M}$ & $\overline{\mathrm{NSW}}$ & NR & LO & 37 & $\mathrm{NC}$ & NSW & NR & $\overline{V L}$ \\
\hline 6 & $\overline{S M}$ & $\overline{\mathrm{NSW}}$ & NSN & LO & 38 & $\mathrm{NC}$ & NSW & $\mathrm{NSN}$ & $\overline{V L}$ \\
\hline 7 & $\overline{\mathrm{SM}}$ & $\overline{\mathrm{NSW}}$ & $\mathrm{NSF}$ & $\overline{\mathrm{LH}}$ & 39 & $\mathrm{NC}$ & NSW & $\mathrm{NSF}$ & $\overline{V L}$ \\
\hline 8 & SM & $\overline{\mathrm{NSW}}$ & FA & LH & 40 & $\mathrm{NC}$ & NSW & FA & LO \\
\hline 9 & SM & $\mathrm{NO}$ & NR & LH & 41 & $\mathrm{NC}$ & $\mathrm{NO}$ & NR & $\mathrm{VL}$ \\
\hline 10 & SM & $\mathrm{NO}$ & NSN & $\mathrm{HG}$ & 42 & $\overline{\mathrm{NC}}$ & $\overline{\mathrm{NO}}$ & $\overline{\mathrm{NSN}}$ & $\overline{\mathrm{LO}}$ \\
\hline 11 & SM & $\mathrm{NO}$ & $\mathrm{NSF}$ & $\mathrm{HG}$ & 43 & $\overline{\mathrm{NC}}$ & $\overline{\mathrm{NO}}$ & $\overline{\mathrm{NSF}}$ & $\overline{\mathrm{LO}}$ \\
\hline 12 & $\overline{\mathrm{SM}}$ & $\mathrm{NO}$ & $\overline{F A}$ & $\overline{\mathrm{HG}}$ & 44 & $\overline{\mathrm{NC}}$ & $\overline{\mathrm{NO}}$ & $\overline{F A}$ & $\overline{\mathrm{LH}}$ \\
\hline 13 & SM & ST & NR & $\mathrm{HG}$ & 45 & $\mathrm{NC}$ & ST & NR & $\overline{\mathrm{LH}}$ \\
\hline 14 & SM & ST & $\overline{\mathrm{NSN}}$ & $\overline{\mathrm{HG}}$ & 46 & $\overline{\mathrm{NC}}$ & ST & $\overline{\mathrm{NSN}}$ & $\overline{\mathrm{LH}}$ \\
\hline 15 & SM & ST & NSF & $\mathrm{HG}$ & 47 & $\mathrm{NC}$ & ST & NSF & $\mathrm{HG}$ \\
\hline 16 & SM & ST & FA & HG & 48 & $\mathrm{NC}$ & ST & FA & HG \\
\hline 17 & $\overline{\mathrm{LC}}$ & $\overline{\mathrm{WK}}$ & $\overline{\mathrm{NR}}$ & $\overline{\mathrm{VL}}$ & 49 & BG & WK & NR & $\overline{\mathrm{VL}}$ \\
\hline 18 & LC & WK & $\mathrm{NSN}$ & $\overline{\mathrm{VL}}$ & 50 & $\overline{B G}$ & WK & NSN & $\overline{V L}$ \\
\hline 19 & $\overline{\mathrm{LC}}$ & WK & NSF & $\mathrm{LO}$ & 51 & $\overline{B G}$ & WK & $\mathrm{NSF}$ & $\overline{V L}$ \\
\hline 20 & LC & WK & FA & LO & 52 & BG & WK & FA & $\overline{V L}$ \\
\hline 21 & $\mathrm{LC}$ & NSW & $\overline{N R}$ & LO & 53 & $\overline{B G}$ & NSW & $\overline{\mathrm{NR}}$ & $\overline{V L}$ \\
\hline 22 & $\overline{\mathrm{LC}}$ & NSW & $\overline{\mathrm{NSN}}$ & LO & 54 & $\overline{B G}$ & NSW & NSN & $\mathrm{VL}$ \\
\hline 23 & $\overline{\mathrm{LC}}$ & NSW & NSF & LO & 55 & BG & NSW & NSF & $\mathrm{VL}$ \\
\hline 24 & LC & NSW & FA & LH & 56 & BG & NSW & FA & $\mathrm{LO}$ \\
\hline 25 & LC & $\mathrm{NO}$ & $\mathrm{NR}$ & LH & 57 & BG & $\mathrm{NO}$ & NR & $\mathrm{VL}$ \\
\hline 26 & LC & $\mathrm{NO}$ & NSN & LH & 58 & $\overline{B G}$ & $\mathrm{NO}$ & NSN & $\mathrm{VL}$ \\
\hline 27 & LC & $\mathrm{NO}$ & $\mathrm{NSF}$ & HG & 59 & $\overline{B G}$ & $\mathrm{NO}$ & NSF & $\mathrm{LO}$ \\
\hline 28 & LC & $\mathrm{NO}$ & FA & $\mathrm{HG}$ & 60 & $\overline{B G}$ & $\mathrm{NO}$ & FA & $\overline{\mathrm{LO}}$ \\
\hline 29 & LC & ST & $\begin{array}{l}\mathrm{NR} \\
\end{array}$ & LH & 61 & $\mathrm{BG}$ & ST & NR & VL \\
\hline 30 & LC & ST & NSN & $\mathrm{HG}$ & 62 & $\mathrm{BG}$ & ST & NSN & $\mathrm{VL}$ \\
\hline 31 & LC & ST & NSF & $\mathrm{HG}$ & 63 & $\overline{B G}$ & ST & NSF & $\mathrm{LO}$ \\
\hline 32 & LC & ST & FA & $\mathrm{HG}$ & 64 & BG & ST & FA & LO \\
\hline
\end{tabular}

The system operation is as follows. First, after receiving the control information from MS, the POTLC check the quality of the signal. If the signal strength is still good enough the handover is not carried out. If the signal strength is lower than a predefined value, then based on CSSP, SSN and $D M B$, the FLC decides whether the handover is necessary or not. If the handover is not necessary the control is returned to the present BS, otherwise another check of the signal strength is carried out in PRTLC and the present signal strength is compared with the previous signal strength. When the present signal strength is lower than the strength of the previous signal, the handover procedure is carried out.

The term sets of CSSP, SSN and DMB are defined respectively as:

$T(C S S P)=\{$ Small, Little Change, No Change, Big $\}$

$$
\begin{aligned}
& =\{S M, L C, N C, B G\} \\
T(S S N) & =\{\text { Weak,Not So Weak, Normal, Strong }\} \\
& =\{\text { WK, NSW,NO,ST }\} \\
T(D M B) & =\{\text { Near, Not So Near, Not So Far, Far }\} \\
& =\{\text { NR, NSN,NSF,FA }\} .
\end{aligned}
$$

The output linguistic parameter $T(H D)$ is defined as $\{$ Very Low, Low, Little High,High $\}=$ $\{V L, L O, L H, H G\}$.

The membership functions of FLC are shown in Fig. 5. The FRB forms a fuzzy set of dimensions $|T(C S S P)| \times$ $|T(S S N)| \times|T(D M B)|$, where $|T(x)|$ is the number of terms on $T(x)$. The FRB is shown in Table 1 and has 64 rules. The control rules have the following form: IF "conditions" THEN "control action". 


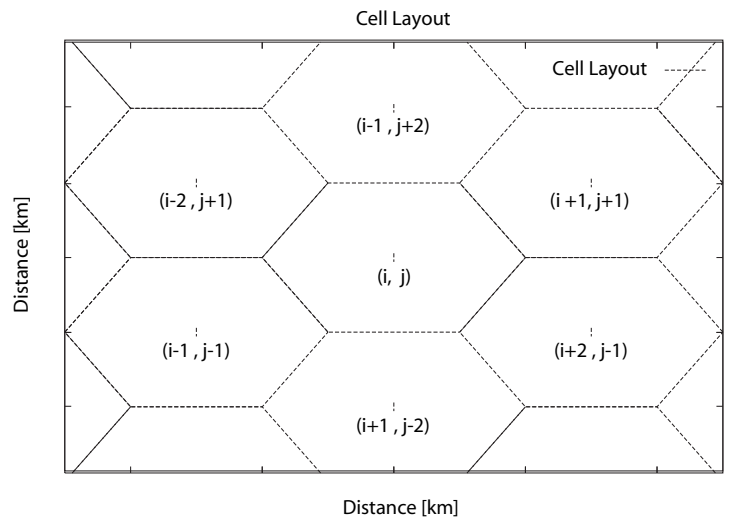

Figure 6. Cell layout.

\section{Simulation Results}

The cell shape is hexagonal and the coordinates of BSs are indicated as shown in Fig. 6. The BS is located in the center of the cell, the transmission antenna power is $10 \mathrm{~W}$, and cell radius is $2 \mathrm{~km}$. In Table 2 are shown the simulation parameters.

In Fig. 7 is showing the walking pattern for a MS when $i$ seed $=100$ and nwalk $=5$, while in Fig. 8 for iseed $=200$ and nwalk $=10$. In Fig. 7, the MS moves in the cells: $(0,0) \rightarrow(2,-1) \rightarrow(0,0) \rightarrow(1,-2)$, while in Fig. 8 in the cells: $(0,0) \rightarrow(-1,2) \rightarrow(-2,1) \rightarrow(-1,2)$. In Fig. 7, the ping-pong effect happens, because the MS is moving in the cells boundary. While in Fig. 8, the handover process is necessary.

In Fig. 9, Fig. 10 and Fig. 11 are showing the received power from the $\mathrm{BS}(0,0), \mathrm{BS}(2,-1)$ Cand $\mathrm{BS}(1,-2)$ when $i$ seed $=200$. We have also the results for $i$ seed $=$ 100 , but for the sake of space will not show in this paper. As can be seen from Fig. 9, when the MS is going far from the BS the received power is decreased, while when the MS is approaching neighbor BS the received power from these BSs is increased (see Fig. 10 and Fig. 11).

For evaluation of the proposed fuzzy-based handover system, we carried out the measurement for 3 points, where the MS is in the boundary of the 3 cells. In Fig. 12 and Fig. 13 are shown the measurement points for $i$ seed $=100$ and iseed $=200$, respectively. In Fig. 12, the handover should not be carried out, because we will have the ping-pong effect, while in Fig. 13 the handover is necessary because the MS is moving inside the neighbor cells.

In our system, we consider that the handover is carried out when the output value is bigger than 0.7. We assume that during the RW for each $10 \mathrm{~km} / \mathrm{h}$ the signal strength is decreased $2 \mathrm{db}$. We carry out 10 times simulations and calculate the average values. The simulation results for iseed $=100$ and iseed $=200$ are shown in Table 3 and
Table 2. Simulation parameters.

\begin{tabular}{|c|c|}
\hline Distribution Law & Gaussian Distribution \\
\hline Number of Walks & $5 \mathrm{C} 10$ \\
\hline Random Types & $100 \mathrm{C} 200$ \\
\hline Cell Radius & $1 \mathrm{kmC} 2 \mathrm{~km}$ \\
\hline Transmission Power & $10 \mathrm{WC} 20 \mathrm{~W}$ \\
\hline Frequency & $2000 \mathrm{MHz}$ \\
\hline Transmission Antenna Beam Tilting & $3^{\circ}$ \\
\hline Transmission Antenna Height & $40 \mathrm{~m}$ \\
\hline Receiving Antenna Height & $1.5 \mathrm{~m}$ \\
\hline Average Value for a Walk & $0.6 \mathrm{~km}$ \\
\hline$n$ & 1.1 \\
\hline
\end{tabular}

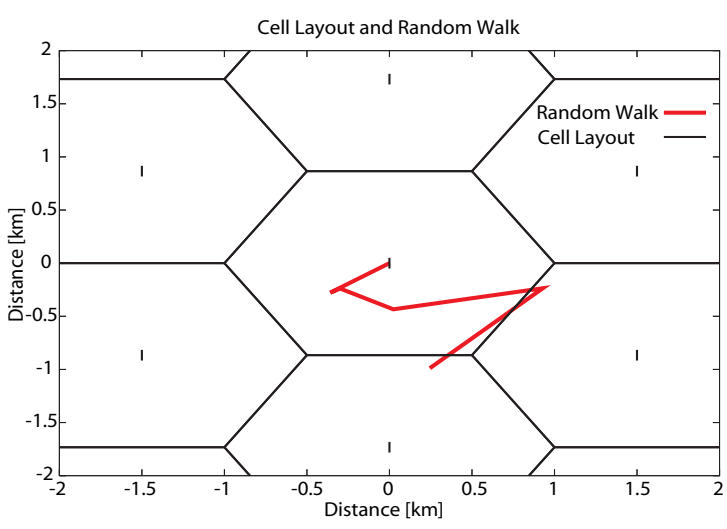

Figure 7. RW pattern for iseed $=100$.

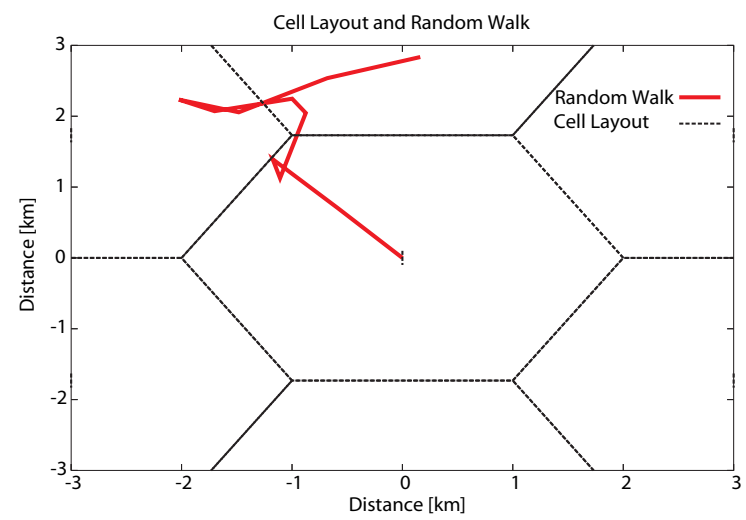

Figure 8. RW pattern for $i$ seed $=200$.

Table 4, respectively.

In the case when $i$ seed $=100$, the MS moves in the boundary of cells. Thus if the handover will be carried out, we will have the ping-pong effect. As shown in Table 3, all the average values are smaller than 0.7 , therefore the proposed system can avoid the ping-pong effect.

In the case when $i$ seed $=200$, the MS is moving inside the neighbor cells, so the handover should be carried out 3 times. In the results of Table 4, the proposed system in all cases has done 3 handovers. This shows that the proposed system has a good handover decision. 
Table 3. Simulation results for $i$ seed $=100$.

\begin{tabular}{|c|c|c|c|c|c|c|}
\hline Measurement Points & Point 1 & \multicolumn{2}{c|}{ Point 2 } & \multicolumn{1}{c|}{ Point 3 } \\
\hline \hline \multicolumn{7}{|c|}{ Speed 0 km/h } \\
\hline CSSP BS & -2.710 & -3.697 & -1.289 & 0.3877 & -1.189 & -1.270 \\
\hline Neighbor BS & -93.36 & -92.49 & -92.77 & -92.77 & -94.01 & -95.28 \\
\hline Distance & 0.8858 & 0.9453 & 0.8684 & 0.8466 & 0.9367 & 1.0183 \\
\hline System Output Value & 0.693 & 0.600 & 0.539 & 0.497 & 0.571 & 0.600 \\
\hline \multicolumn{7}{|c|}{ Speed 10 km/h } \\
\hline CSSP BS & -2.710 & -3.697 & -1.289 & 0.3877 & -1.189 & -1.270 \\
\hline Neighbor BS & -95.36 & -94.49 & -94.77 & -94.77 & -96.01 & -97.28 \\
\hline Distance & 0.8858 & 0.9427 & 0.8684 & 0.8466 & 0.9367 & 1.0183 \\
\hline System Output Value & 0.693 & 0.600 & 0.583 & 0.542 & 0.600 & 0.618 \\
\hline \multicolumn{7}{|c|}{ Speed 20 km/h } \\
\hline CSSP BS & -2.710 & -3.697 & -1.289 & 0.3877 & -1.189 & -1.270 \\
\hline Neighbor BS & -97.36 & -96.49 & -96.77 & -96.77 & -98.01 & -99.28 \\
\hline Distance & 0.8858 & 0.9401 & 0.8684 & 0.8466 & 0.9367 & 1.0183 \\
\hline System Output Value & 0.693 & 0.600 & 0.614 & 0.574 & 0.624 & 0.640 \\
\hline \multicolumn{7}{|c|}{ Speed 30 km/h } \\
\hline CSSP BS & -2.710 & -3.697 & -1.289 & 0.3877 & -1.189 & -1.270 \\
\hline Neighbor BS & -99.36 & -98.49 & -98.77 & -98.77 & -100.0 & -101.3 \\
\hline Distance & 0.8858 & 0.9376 & 0.8684 & 0.8466 & 0.9367 & 1.0183 \\
\hline System Output Value & 0.693 & 0.600 & 0.632 & 0.584 & 0.645 & 0.657 \\
\hline \multicolumn{7}{|c|}{ Speed 40 km/h } \\
\hline CSSP BS & -2.710 & -3.697 & -1.289 & 0.3877 & -1.189 & -1.270 \\
\hline Neighbor BS & -101.4 & -100.5 & -100.8 & -100.8 & -102.0 & -103.3 \\
\hline Distance & 0.8858 & 0.9351 & 0.8684 & 0.8466 & 0.9367 & 1.0183 \\
\hline System Output Value & 0.693 & 0.600 & 0.631 & 0.582 & 0.656 & 0.662 \\
\hline \multicolumn{7}{|c|}{ Speed 50 km/h } \\
\hline CSSP BS & -2.710 & -3.697 & -1.289 & 0.3877 & -1.189 & -1.270 \\
\hline Neighbor BS & -103.4 & -102.5 & -102.8 & -102.8 & -104.0 & -105.3 \\
\hline Distance & 0.8858 & 0.9327 & 0.8684 & 0.8466 & 0.9367 & 1.0183 \\
\hline System Output Value & 0.693 & 0.600 & 0.631 & 0.582 & 0.656 & 0.663 \\
\hline
\end{tabular}

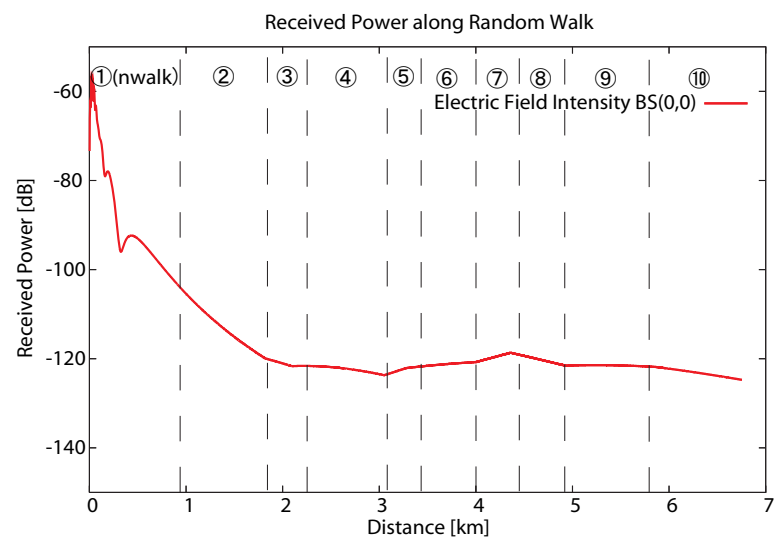

Figure 9. Received power from $B S(0,0)$ $($ iseed $=200)$.

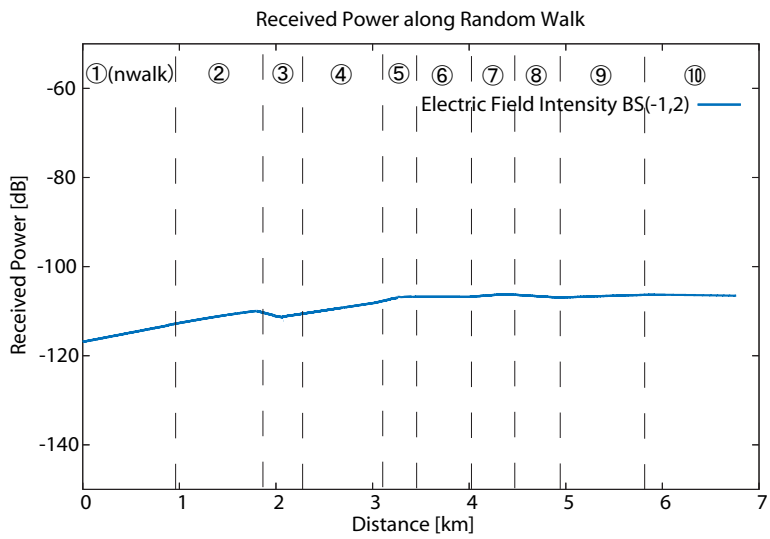

Figure 10. Received power from BS(-1,2) (iseed $=200)$. 
Table 4. Simulation results for $i$ seed $=200$.

\begin{tabular}{|c|c|c|c|c|c|c|}
\hline Measurement Points & \multicolumn{2}{|c|}{ Point 1 } & \multicolumn{2}{c|}{ Point 2 } & \multicolumn{2}{c|}{ Point 3 } \\
\hline \hline CSSP BS & -2.0149 & -3.4731 & -2.1681 & -3.7153 & -7.1891 & -7.9733 \\
\hline Neighbor BS & -105.55 & -102.07 & -103.52 & -96.763 & -103.85 & -88.422 \\
\hline Distance & 1.9597 & 2.4628 & 1.8367 & 2.3453 & 1.8021 & 3.0449 \\
\hline System Output Value & 0.645 & $\mathbf{0 . 7 4 5}$ & 0.634 & $\mathbf{0 . 7 4 0}$ & 0.692 & $\mathbf{0 . 7 3 0}$ \\
\hline \multicolumn{7}{|c|}{ Speed 10 km/h } \\
\hline CSSP BS & -2.0149 & -3.4731 & -2.1681 & -3.7153 & -7.1891 & -7.9733 \\
\hline Neighbor BS & -107.55 & -104.07 & -105.52 & -98.763 & -105.85 & -90.442 \\
\hline Distance & 1.9597 & 2.4628 & 1.8367 & 2.3453 & 1.8021 & 3.0449 \\
\hline System Output Value & 0.632 & $\mathbf{0 . 7 8 0}$ & 0.634 & $\mathbf{0 . 7 1 0}$ & 0.671 & $\mathbf{0 . 7 3 0}$ \\
\hline \multicolumn{7}{|c|}{ Speed $20 \mathrm{~km} / \mathrm{h}$} \\
\hline CSSP BS & -2.0149 & -3.4731 & -2.1681 & -3.7153 & -7.1891 & -7.9733 \\
\hline Neighbor BS & -109.55 & -106.07 & -107.52 & -100.76 & -107.85 & -92.422 \\
\hline Distance & 1.9597 & 2.4628 & 1.8367 & 2.3453 & 1.8021 & 3.0449 \\
\hline System Output Value & 0.616 & $\mathbf{0 . 7 7 7}$ & 0.620 & $\mathbf{0 . 7 2 6}$ & 0.633 & $\mathbf{0 . 7 3 0}$ \\
\hline \multicolumn{7}{|c|}{ Speed 30 km/h } \\
\hline CSSP BS & -2.0149 & -3.4731 & -2.1681 & -3.7153 & -7.1891 & -7.9733 \\
\hline Neighbor BS & -111.55 & -108.07 & -109.52 & -102.76 & -109.85 & -94.422 \\
\hline Distance & 1.9597 & 2.4628 & 1.8367 & 2.3453 & 1.8021 & 3.0449 \\
\hline System Output Value & 0.596 & $\mathbf{0 . 7 4 3}$ & 0.597 & $\mathbf{0 . 7 5 6}$ & 0.606 & $\mathbf{0 . 7 3 0}$ \\
\hline \multicolumn{7}{|c|}{ Speed 40 km/h } \\
\hline CSSP BS & -2.0149 & -3.4731 & -2.1681 & -3.7153 & -7.1891 & -7.9733 \\
\hline Neighbor BS & -113.55 & -110.07 & -111.52 & -104.76 & -111.85 & -96.422 \\
\hline Distance & 0.3536 & 0.4821 & 0.6824 & 0.9047 & 1.3158 & 1.4976 \\
\hline System Output Value & 0.576 & $\mathbf{0 . 7 1 5}$ & 0.574 & $\mathbf{0 . 7 9 4}$ & 0.591 & $\mathbf{0 . 7 2 8}$ \\
\hline \multicolumn{7}{|c|}{ Speed 50 km/h } \\
\hline CSSP BS & -2.0149 & -3.4731 & -2.1681 & -3.7153 & -7.1891 & -7.9733 \\
\hline Neighbor BS & -115.55 & -112.07 & -113.52 & -106.76 & -113.85 & -98.422 \\
\hline Distance & 0.3536 & 0.4821 & 0.6824 & 0.9047 & 1.3158 & 1.4976 \\
\hline System Output Value & 0.545 & $\mathbf{0 . 7 0 3}$ & 0.553 & $\mathbf{0 . 7 1 3}$ & 0.579 & $\mathbf{0 . 7 0 3}$ \\
\hline
\end{tabular}

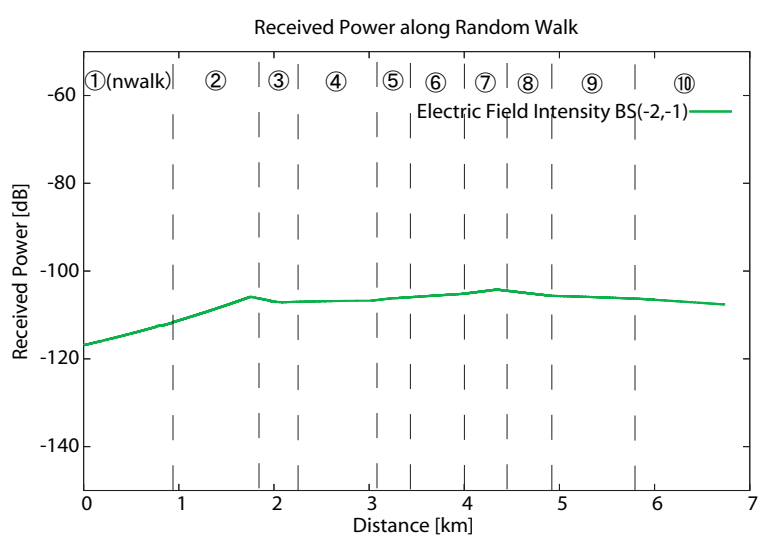

Figure 11. Received power from BS(-2,1) $($ iseed $=200)$.

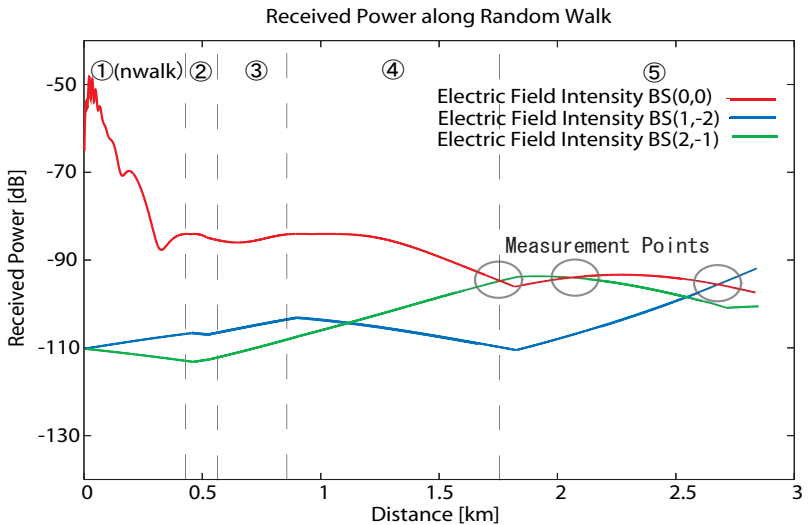

Figure 12. 3 measurement points for $i$ seed $=$ 100. 


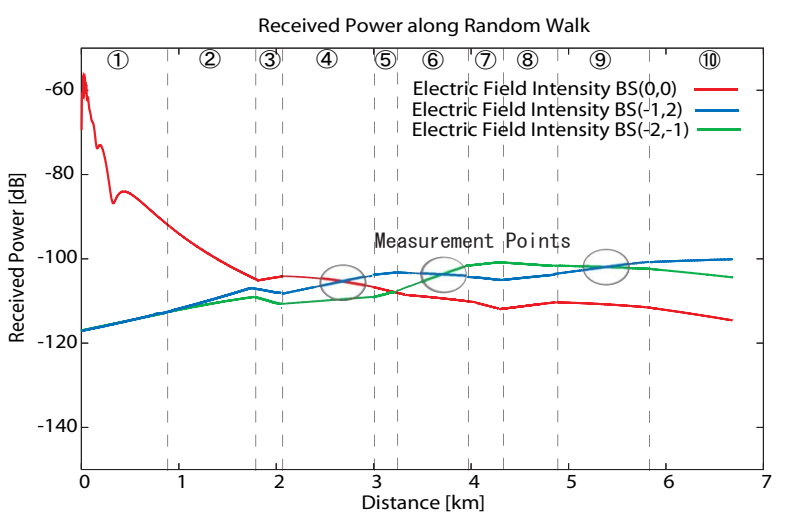

Figure 13. 3 measurement points for $i$ seed $=$ 200.

\section{Conclusions}

Many investigations have addressed handover algorithms for cellular communication systems. However, it is essentially complex to make handover decision considering multiple criteria. Sometimes, the trade-off of some criteria should be considered.

Because of large-scale and small-scale fades are frequently encountered in mobile environment, it is very difficult for handover algorithm to make an accurate and timely decision. Handover algorithms operating in real time have to make decisions without the luxury of repeated uncorrelated measurements. Some of handover criteria information can be inherently imprecise, or the precise information is difficult to obtain. For this reason, we proposed a FL-based approach, which can operate with imprecision data and can model nonlinear functions.

In this paper, we proposed a handover system using RW model and FL. The proposed system can avoid the pingpong effect and has a good handover decision.

In the future, we would like to compare the performance of the proposed system with other non-fuzzy-based handover algorithms.

\section{References}

[1] R. Berezdivin, R. Breining, R. Topp, "NextGeneration Wireless Communication Concepts and Technologies", IEEE Communication Magazine, Vol. 40, No. 3, pp. 108-116, 2002.

[2] Y. Guo, H. Chaskar, "Class-Based Quality of Service over Air Interfaces in 4G Mobile Networks", IEEE Communication Magazine, Vol. 40, No. 3, pp. 132$137,2002$.

[3] W. Wang, X. Wang, A.A. Nilsson, ”Energy-Efficient Bandwidth Allocation in Wireless Networks: Algorithms, Analysis, and Simulations", IEEE Transactions on Wireless Communications, Vol. 5, No. 5, pp. 1103-1114, 2006.
[4] Y. Fang, Y. Zhang, "Call Admission Control Schemes and Performance Analysis in Wireless Mobile Networks", IEEE Transactions on Vehicular Technology, Vol. 51, No. 2, pp. 371-382, 2002.

[5] K. D. Wong, D.C. Cox, "A Pattern Recognition System for Handoff Algorithms", IEEE J-SAC, Vol. 18, No. 7, pp. 1301-1312, 2000.

[6] S. Kovvuri, V. Pandey, D. Ghosal, B. Mukherjee, D. Sarkar, "A Call-Admission Control (CAC) Algorithm for Providing Guaranteed QoS in Cellular Networks", International Journal of Wireless Information Networks, Vol. 10, No. 2, pp. 73-85, 2003.

[7] H.P. Lin, R.T. Juang, D.B. Lin, "Validation of an Improved Location-Based Handover Algorithm Using GSM Measurement Data", IEEE Transactions on Mobile Computing, Vol. 4, No. 5, pp. 530-536, 2005.

[8] P. Fiengo, G. Giambene, E. Trentin, "Neural-based Downlink Scheduling Algorithm for Broadband Wireless Networks", Computer Communication, Vol. 30, No. 2, pp. 207-218, 2007.

[9] L. Barolli, A. Koyama, T. Suganuma, N. Shiratori, "GAMAN: A GA Based QoS Routing Method for Mobile Ad-hoc Networks", Journal of Interconnection Networks (JOIN), Vol. 4, No. 3, pp. 251-270, 2003.

[10] T. Onel, C. Ersoy, E. Cayirci, ”A Fuzzy Inference System for the Handoff Decision Algorithms in the Virtual Cell Layout Base Tactical Communications System", IEEE Military Communications Conference (MILCOM-2002), Vol. 1, pp. 436-441, 2002.

[11] P. M. L. Chan, R. E. Sheriff, Y.F. Hu, P. Conforto, C. Tocci, "Mobility Management Incorporating Fuzzy Logic for a Heterogeneous IP Environment", IEEE Communications Magazine, Vol. 39, No. 12, pp. 42$51,2001$.

[12] S. Mohanty, I.F. Akyildiz, "A Cross-Layer (Layer 2+3) Handoff Management Protocol for NextGeneration Wireless Systems", IEEE Transactions on Mobile Computing, Vol. 5, No. 10, pp. 1347-1360, 2006.

[13] F. Yu, Y. Krishamurthy, "Optimal Joint Session Admission Control in Integrated WLAN and CDMA Cellular Networks with Vertical Handoff", IEEE Transactions on Mobile Computing, Vol. 6, No. 1, pp. 126139, 2007.

[14] D. Dubois, H. Prade, R. Yager, (Eds.), "Fuzzy Sets for Intelligent Systems", Morgan Kaufman Publishers, 1993. 